\title{
ULTRALOW BEAM DIVERGENCE AND INCREASED LATERAL BRIGHTNESS IN OPTICALLY PUMPED MIDINFRARED LASER (POSTPRINT)
}

Ron Kaspi, et al.

1 April 2012

Technical Paper

APPROVED FOR PUBLIC RELEASE; DISTRIBUTION IS UNLIMITED.

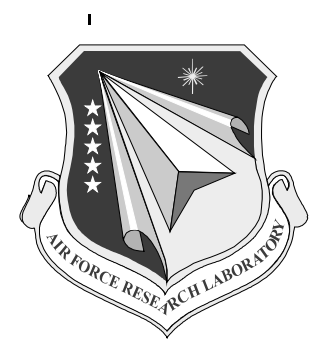

AIR FORCE RESEARCH LABORATORY Directed Energy Directorate 3550 Aberdeen Ave SE AIR FORCE MATERIEL COMMAND KIRTLAND AIR FORCE BASE, NM 87117-5776 


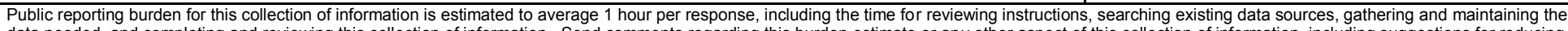

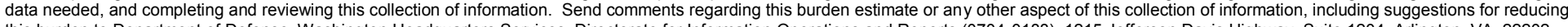

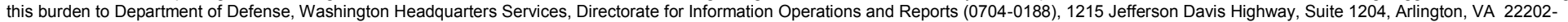

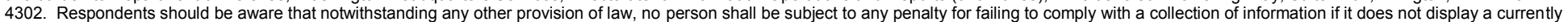
valid OMB control number. PLEASE DO NOT RETURN YOUR FORM TO THE ABOVE ADDRESS.

\begin{tabular}{l|l|l} 
1. REPORT DATE (DD-MM- $Y Y Y Y)$ & 2. REPORT TYPE & 3. DATES COVERED (FrOm - TO)
\end{tabular}

\begin{tabular}{l|l}
$1 / 04 / 2012$ & Technical Paper
\end{tabular}

4. TITLE AND SUBTITLE

Ultralow Beam Divergence and Increased Lateral Brightness in Optically

Pumped Midinfrared Laser (Postprint)

1Apr 2012-1 Apr 2012

5a. CONTRACTNUMBER

CRADA 2006-AFRLDE03

5b. GRANT NUMBER

5c. PROGRAM ELEMENT NUMBER

6. AUTHOR(S)

5d. PROJECT NUMBER

Ron Kaspi, Michael L. Tilton, Gregory C. Dente, Joseph R. Chavez, Andrew Ongstad

5e. TASK NUMBER

5f. WORK UNIT NUMBER

D010

7. PERFORMING ORGANIZATION NAME(S) AND ADDRESS(ES)

Air Force Research Laboratory

8. PERFORMING ORGANIZATION REPORT NUMBER

3550 Abderdeen Ave SE

Kirtland AFB, NM

$87117-5776$

9. SPONSORING / MONITORING AGENCY NAME(S) AND ADDRESS(ES)

Air Force Research Laboratory

3550 Abderdeen Ave SE

Kirtland AFB, NM

$87117-5776$

10. SPONSOR/MONITOR'S ACRONYM(S)

AFRL/RDLT

11. SPONSOR/MONITOR'S REPORT

NUMBER(S)

12. DISTRIBUTION / AVAILABILITY STATEMENT

AFRL-RD-PS-TP-2016-0002

Approved for public release: distribution unlimited. 377ABW-2011-1428; 4 Oct 2011.

\section{SUPPLEMENTARY NOTES}

Accepted for publication in the IEEE Photonics Technology Letters, Vol. 24, No. 7, April 1, 2012. "'Government Purpose Rights"

\section{ABSTRACT}

An optically pumped edge-emitting semiconductor laser emitting near $4.1 \mu \mathrm{m}$ was designed with weak transverse mode confinement resulting in an exceptionally large transverse optical mode size. Consequently, a laser device is reported that exhibits a fast-axis divergence angle of $\sim 4.2^{\circ}$ full-width at half-maximum (FWHM). More notably, a large reduction of the lateral axis divergence is also observed as a result of the increased lateral coherency. The reduced confinement factor and differential gain serves to suppress lateral filamentation. Despite the higher threshold and lower power, a lateral divergence angle of $\sim 3.2^{\circ} \mathrm{FWHM}$ and increased lateral brightness is achieved.

\section{SUBJECT TERMS}

Antimonide laser, low beam divergence, midinfrared laser, optically pumped laser, type-II quantum wells

\begin{tabular}{|c|c|c|c|c|c|}
\hline 16. SECURITY Cl & IFICATION OF: & & 17. LIMITATION & 18. NUMBER & 19a. NAME OF RESPONSIBLE PERSON \\
\hline $\begin{array}{l}\text { a. REPORT } \\
\text { Unclassified }\end{array}$ & $\begin{array}{l}\text { b. ABSTRACT } \\
\text { Unclassified }\end{array}$ & $\begin{array}{l}\text { c. THIS PAGE } \\
\text { Unclassified }\end{array}$ & SAR & 6 & $\begin{array}{l}\text { 19b. TELEPHONE NUMBER (include area } \\
\text { code) } \\
505-846-5879\end{array}$ \\
\hline
\end{tabular}




\title{
Ultralow Beam Divergence and Increased Lateral Brightness in Optically Pumped Midinfrared Laser
}

\author{
Ron Kaspi, Michael L. Tilton, Gregory C. Dente, Joseph R. Chavez, and Andrew P. Ongstad
}

\begin{abstract}
An optically pumped edge-emitting semiconductor laser emitting near $4.1 \mu \mathrm{m}$ was designed with weak transverse mode confinement resulting in an exceptionally large transverse optical mode size. Consequently, a laser device is reported that exhibits a fast-axis divergence angle of $\sim 4.2^{\circ}$ full-width at half-maximum (FWHM). More notably, a large reduction of the lateral axis divergence is also observed as a result of the increased lateral coherency. The reduced confinement factor and differential gain serves to suppress lateral filamentation. Despite the higher threshold and lower power, a lateral divergence angle of $\sim 3.2^{\circ}$ FWHM and increased lateral brightness is achieved.
\end{abstract}

Index Terms-Antimonide laser, low beam divergence, midinfrared laser, optically pumped laser, type-II quantum wells.

\section{INTRODUCTION}

$\mathbf{I}$ $\mathrm{N}$ EPITAXIALLY grown edge-emitting semiconductor lasers, the waveguide is typically engineered to provide a large modal overlap with the gain region so that low threshold and high slope efficiencies can be achieved. As the gain region is contained within the epitaxial growth, the transverse mode of the device is correspondingly thin, typically only a few micrometers. In a $\mathrm{p}-\mathrm{i}-\mathrm{n}$ junction diode laser, the transverse mode size is further restricted by the necessity to flow current across this junction while minimizing resistive heating. Diode laser designs must often mitigate between minimizing the thickness of the undoped gain region to reduce the operating voltage, and maximizing the undoped gain region to reduce waveguide losses arising from the penetration of the mode into doped clad regions. These considerations result in semiconductor edge-emitting lasers that exhibit a relatively large transverse axis divergence angle when compared to other large mode-size devices such as solid-state disk or fiber lasers. The fast-axis beam divergence, whether from a 980nm semiconductor laser diode, or a midinfrared quantum cascade laser, typically ranges between 20 to 40 degrees as measured at the full-width at half-maximum (FWHM) point, [1] unless additional beam shaping features such as plasmonic collimation gratings are added [2]. Such a large divergence

Manuscript received October 18, 2011; revised November 17, 2011; accepted January 7, 2012. Date of publication January 16, 2012; date of current version March 16, 2012. This work was supported in part by the Air Force Office of Scientific Research.

The authors are with the Air Force Research Laboratory, Albuquerque, NM 87117 USA (e-mail: Ron.Kaspi@kirtland.af.mil; michael.tilton@kirtland.af.mil; gcdente@gmail.com; joseph.chavez2@ kirtland.af.mil; andrew.ongstad@kirtland.af.mil).

Color versions of one or more of the figures in this letter are available online at http://ieeexplore.ieee.org.

Digital Object Identifier 10.1109/LPT.2012.2184279 makes it difficult to capture and manipulate the output beam, and is a distinct disadvantage of semiconductor lasers.

In optically pumped semiconductor laser designs, waveguide clad layers do not need to be intentionally doped. As a result, there is additional flexibility in the design of the waveguide without fear of extensive waveguide loss in the clad regions. In this letter, we demonstrate that an optically pumped device can be engineered to deliberately extend the transverse mode a very large distance into the substrate. As a result, devices with ultralow fast-axis divergence angles can be produced. As an important added benefit of the reduced overlap with the gain region, we also find that the lateral spatial coherence of the device is also greatly improved, resulting in ultralow lateral divergence from a broad area optically pumped laser.

\section{EXPERIMENT}

The semiconductor heterostructure design used in this experiment is similar to previously reported edge-emitting optically pumped semiconductor lasers (OPSL) grown on GaSb:Te (001) substrates. [3] The heterostructure consists of a $0.2-\mu \mathrm{m}$-thick GaSb buffer layer, followed by a $\sim 2-\mu \mathrm{m}$-thick lattice-matched $\mathrm{In}_{0.16} \mathrm{Ga}_{0.84} \mathrm{As}_{y} \mathrm{Sb}_{1-y}$ waveguide core region containing fourteen $\mathrm{InAs} / \mathrm{In}_{0.4} \mathrm{Ga}_{0.6} \mathrm{Sb} / \mathrm{InAs}$ type-II quantum wells to provide gain. This is followed by a $\sim 4-\mu \mathrm{m}$-thick GaSb undoped top clad layer. Finally, a $0.5-\mu \mathrm{m}$-thick nearlylattice-matched low-index $\mathrm{AlAs}_{1-y} \mathrm{Sb}_{y}$ cap layer is grown to prohibit the transverse mode from extending beyond the device when it is in the epi-down configuration. The quaternary alloy of the waveguide is designed to absorb the $1.9-\mu \mathrm{m}$ pump radiation to create electron-hole pairs. These carriers recombine in the quantum wells to produce emission near $4.1 \mu \mathrm{m}$. The transverse mode is only weakly confined by the GaSb substrate at the bottom and the GaSb top clad, with refractive indexes only slightly lower than that of the waveguide core.

The experiment was designed to manipulate the extension of the transverse mode into the GaSb substrate by using three different GaSb:Te substrates, each with a different nominal doping concentration. The refractive index of the $\mathrm{GaSb}$ substrate is expected to decrease as the tellurium dopant concentration is increased [4]. Laser-A was grown on a substrate with a nominal carrier concentration of $1.4-1.6 \times 10^{18} / \mathrm{cm}^{3}$ measured at room temperature. For Laser-B, the carrier concentration was estimated to be $2.6-2.8 \times 10^{17} / \mathrm{cm}^{3}$. Finally for Laser$\mathrm{C}$, the nominal carrier concentration was $1-2 \times 10^{16} / \mathrm{cm}^{3}$. 


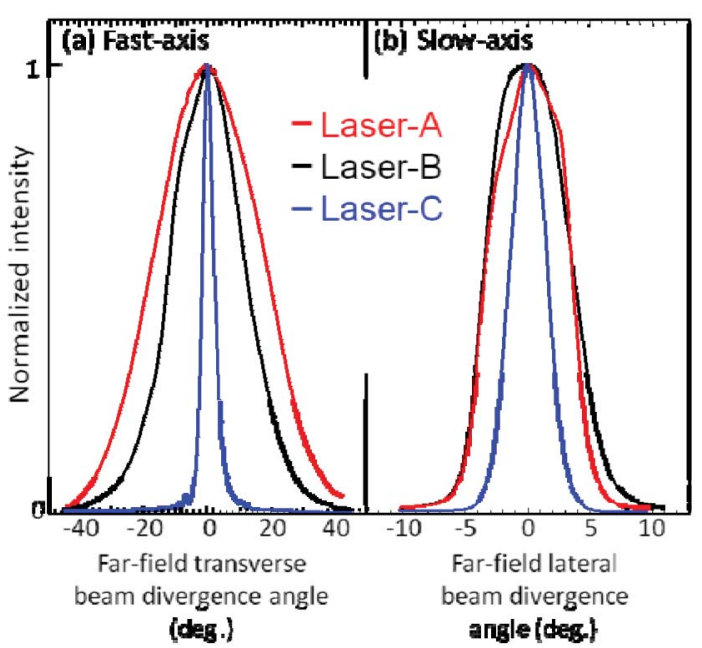

Fig. 1. (a) Far-field transverse beam profile comparison between Laser-A, $-B$, and -C. (b) Far-field lateral-beam profile comparison between Laser-A, $-\mathrm{B}$, and $-\mathrm{C}$.

The laser structure was grown simultaneously on all three wafers, and thus identical. It is expected that the transverse mode will be most confined and least extended in Laser-A, and the least confined and most extended in Laser-C.

Devices were lapped and polished to a nominal thickness of 120-microns, cleaved to a $4 \mathrm{~mm}$ cavity-length, and mounted epi-down for characterization at $80 \mathrm{~K}$. The width of the gainguided device was $\sim 250$-microns, defined by the width of the pump stripe. The $1.9-\mu \mathrm{m}$ pump radiation is not absorbed by the GaSb substrate, thus making epi-down mounting possible. The output power, spectra, and far-field characteristics were measured using $32-\mu$ sec pulses at $1 \%$ duty-cycle.

\section{RESULTS}

Fast axis beam profile measurements from all three devices collected at the highest pump power available are presented in Figure 1(a). Laser-A exhibits a divergence angle of $\sim 39$ degrees as measured at the FWHM. Laser-B exhibits a reduced fast-axis divergence of $\sim 26$ degrees, consistent with a larger transverse mode. In Laser-C, we observe a divergence angle of only $\sim 4.2$ degrees FWHM, confirming a very large transverse mode size.

Slow-axis beam profile measurements are presented in Figure 1(b). Given that these are broad-area devices, this divergence angle is primarily determined by the dimension of the lateral filaments that are formed. Laser-A and Laser-B exhibit a similar lateral beam divergence of $\sim 7.5$ degrees at FWHM, considerably smaller than what is typically observed in broadarea semiconductor diode lasers [1]. Such optically pumped devices were previously shown to provide some protection against filament formation due to a very small line-width enhancement factor $\alpha$, typically $\sim 1$ [5]. In contrast, Laser$\mathrm{C}$ exhibits a lateral beam divergence of only $\sim 3.2$ degrees at FWHM, indicating a further increase in filament size and improved lateral coherency. Evidently, the much larger transverse mode size, along with the corresponding reduction of the confinement factor, $\Gamma$, resulted in a substantial increase

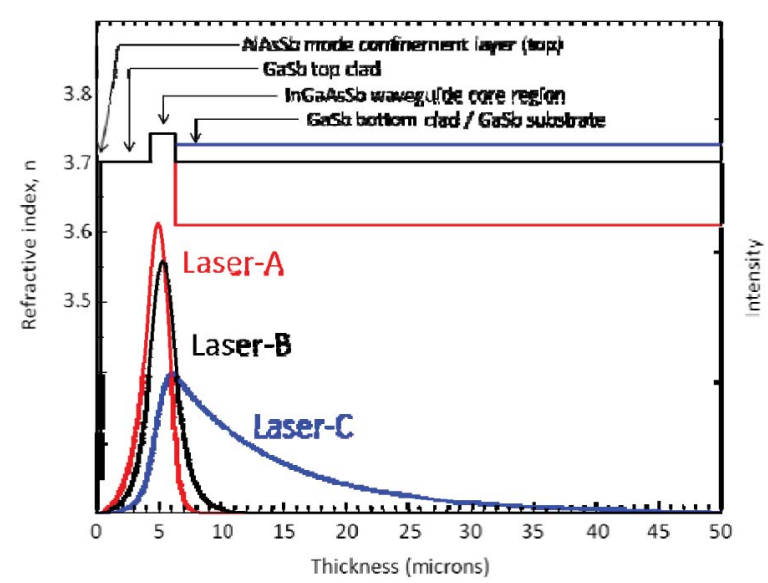

Fig. 2. Calculated transverse modes generated using the substrate refractive index as a fitting parameter to the observed far-field profile. Also shown is the refractive index variation with thickness for Laser-A, $-\mathrm{B}$, and $-\mathrm{C}$.

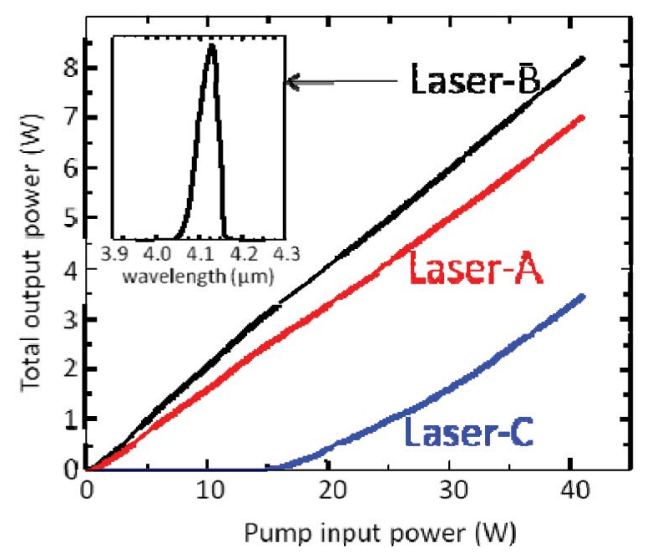

Fig. 3. Input power versus total output power measured at $80 \mathrm{~K}$ from Laser-A, -B, and -C. Emission spectrum from Laser-B is shown in the inset.

of the coherency of the lateral beam in these broad area devices.

Using a complex waveguide model, the refractive index of the different substrates was used as the fitting parameter to match the far-field profiles and estimate the extent of the transverse mode. A refractive index value of $\mathrm{n} \sim 3.74$ at $80 \mathrm{~K}$ was assumed for the waveguide core region. For the substrates of Lasers-A, $-\mathrm{B}$ and $-\mathrm{C}$, a refractive index of $\mathrm{n} \sim 3.61, \mathrm{n} \sim 3.70$, and $\mathrm{n} \sim 3.725$, produced good fits, respectively. The calculated transverse modes are shown in Figure 2. The transverse mode in Laser-C likely reaches approximately 50 micrometers into the substrate due to the extremely low index confinement provided by the waveguide region. We also calculated a reduced core region confinement factor, $\Gamma$, in Laser-C, that is approximately four times smaller than that in Laser-B.

With a large reduction in $\Gamma$, we can expect Laser-C to exhibit increased threshold power relative to Laser-A and Laser-B. The power-power curves for all three devices are shown in Figure 3, and this is indeed the case. The threshold power in both Laser-A and Laser-B is approximately $\sim 150 \mathrm{~W} / \mathrm{cm}^{2}$, whereas the threshold power in Laser-C is 
TABLE I

LASER CHARACTERISTICS

\begin{tabular}{|l|c|c|c|c|c|l|}
\hline & $\theta_{\perp}$ & $\theta_{=}$ & $d_{\perp}$ & $d_{=}$ & $\begin{array}{l}\text { Output } \\
\text { Power }\end{array}$ & $\begin{array}{l}\text { Brightness } \\
(\mathrm{MW} / \\
\left.\mathrm{cm}^{2} \text {-sr }\right)\end{array}$ \\
\hline Laser-A & $39^{\circ}$ & $7.5^{\circ}$ & $7.5-\mu \mathrm{m}$ & $250-\mu \mathrm{m}$ & $7.0 \mathrm{~W}$ & 1.0 \\
\hline Laser-B & $26^{\circ}$ & $7.5^{\circ}$ & $8.0-\mu \mathrm{m}$ & $250-\mu \mathrm{m}$ & $8.2 \mathrm{~W}$ & 1.2 \\
\hline Laser-C & $4.2^{\circ}$ & $3.2^{\circ}$ & $50-\mu \mathrm{m}$ & $250-\mu \mathrm{m}$ & $3.5 \mathrm{~W}$ & 2.5 \\
\hline
\end{tabular}

$\sim 1.6 \mathrm{~kW} / \mathrm{cm}^{2}$. Laser-B exhibits the highest total output power, $\sim 8.2$ Watts from two facets at $\sim 42 \mathrm{~W}$ of input power, the maximum available. Laser-A produced $\sim 7$ Watts of output power due to the slightly lower slope efficiency, presumably as a result of the increased free-carrier losses in the higher doped substrate. Due to the much larger threshold power and slightly lower slope efficiency, laser-C was only able to produce $\sim 3.5 \mathrm{~W}$ at the maximum pump power, albeit with increased lateral coherency. The brightness for a diode laser can be defined as:

$$
B=\frac{\text { Output Power }}{\pi^{2} \cdot\left(B P P_{\perp}^{2}+B P P_{=}^{2}\right)},
$$

where the Beam Parameter Products (BPP's) in the two orthogonal directions (fast and slow axis) are defined as:

$$
\begin{aligned}
& B P P_{\perp}=\left(\frac{\theta_{\perp}}{2}\right) \cdot\left(\frac{d_{\perp}}{2}\right) \\
& B P P_{=}=\left(\frac{\theta_{\bar{E}}}{2}\right) \cdot\left(\frac{d_{\bar{E}}}{2}\right)
\end{aligned}
$$

$\theta$ and $d$ are the full angular divergences and beam diameters in the respective perpendicular and parallel directions. We can calculate the relative brightness of Laser-B and Laser$\mathrm{C}$, operating under identical conditions, at the maximum input pump power of $\sim 42 \mathrm{~W}$. Table I lists the measured and calculated characteristics for each of the lasers.

Laser-B's brightness is estimated to be $\sim 1.2 \mathrm{MW} / \mathrm{cm}^{2}$ sr. In contrast, Laser-C's brightness is estimated to be $\sim 2.5 \mathrm{MW} / \mathrm{cm}^{2}$-sr. Laser-C is capable of producing more than twice the brightness of Laser-B despite its lower output power. This is a direct result of the increased lateral coherence. The fast-axis emission remains near-diffraction limited despite the difference in divergence angle.

The improved lateral coherency and suppressed filament formation in broad-area lasers with extended transverse modes and reduced modal gain overlap can be understood by analyzing the carrier-induced filament formation process near steady-state [6]. The analysis shows that filament gain can be reduced when the line-width enhancement factor, $(\alpha)$, is minimized, when the internal losses, $(a)$, are reduced, or alternatively when the saturation intensity, $\left(I_{S}\right)$, is increased. Because the epitaxial structures were equivalent in this letter, we can expect that $\alpha$ and $a$ are similar.
With the carriers clamped at threshold, the expression for $I_{S}$ is given by:

$$
I_{s}=\frac{\eta_{i} V_{g a p}}{\Gamma \frac{\partial G}{\partial J}}
$$

where $\eta_{i}$ is the injection efficiency, $G$ is the gain, $J$ is the current density, and $V_{g a p}$ is the gap voltage. When the confinement factor $\Gamma$ in Laser-C is reduced, the threshold gain is increased, resulting also in a reduction in the differential gain. Through the inverse dependence of $I_{s}$ on both $\Gamma$ and $\partial \mathrm{G} / \partial \mathrm{J}$, the extended transverse mode will have the effect of increasing the saturation intensity, thus reducing the filament gain. Laser-C is therefore an embodiment of one of the design principles needed to reduce the tendency for lateral filamentation in a broad area laser. [6]

In addition to the nearly cylindrical output beam with very low divergence that is easier to capture and manipulate, and the improved lateral coherency which leads to increased brightness, there are two other good reasons to use a laser designed to support an extended transverse mode. One of these is the reduced photon flux at the facet which will protect against possible heating and facet damage. The other is the fact that external coupling to the laser mode is made easier as a result of the large mode size.

\section{CONCLUSION}

We demonstrate that the transverse mode size can be deliberately extended in an optically pumped design to produce semiconductor edge-emitting lasers with ultralow fast-axis divergence. The resulting increase in threshold density and lower output power does not necessarily result in reduced brightness. This is because an additional benefit of the reduced confinement factor is the suppression of lateral filaments due to the increased saturation intensity. These design principles, put forward in reference 6 , are used here to produce midinfrared lasers with increased brightness.

\section{REFERENCES}

[1] n-Light Corporation 2011 Diode Specifications [Online]. Available: http://www.nlight.net/diodes/details/3 $\sim$ Single-Emitter-Diode-LaserDevices-non-visible

[2] N. Yu, et al., "Quantum cascade lasers with integrated plasmonic antenna-array collimators," Opt. Express, vol. 16, no. 24, pp. 1944719461, 2008.

[3] R. Kaspi, A. P. Ongstad, G. C. Dente, J. R. Chavez, M. L. Tilton, and D. M. Gianardi, "High performance optically pumped antimonide lasers operating in the 2.4-9.3 $\mu \mathrm{m}$ wavelength range," Appl. Phys. Lett., vol. 88, no. 4, pp. 041122-1-041122-3, Jan. 2006.

[4] P. P. Paskov, "Refractive indices of InSb, InAs, GaSb, InAs $\mathrm{Sb}_{1-x}$ and $\mathrm{In}_{1-x} \mathrm{Ga}_{x} \mathrm{Sb}$ : Effects of free carriers," J. Appl. Phys., vol. 81, no. 4, pp. 1890-1898, Feb. 1997.

[5] A. P. Ongstad, G. C. Dente, M. L. Tilton, R. Kaspi, and R. Chavez, "The antiguiding parameter in mid-infrared optically pumped semiconductor lasers," Appl. Phys. Lett., vol. 97, pp. 133-134, Sep. 2010.

[6] G. C. Dente, "Low confinement factors for suppressed filaments in semiconductor lasers," J. Quantum Electron., vol. 37, no. 12, pp. 16501653, Dec. 2001 


\section{DISTRIBUTION LIST}

\section{DTIC/OCP}

8725 John J. Kingman Rd, Suite 0944

Ft Belvoir, VA 22060-6218

1 cy

AFRL/RVIL

Kirtland AFB, NM 87117-5776

1 cy

Ron Kaspi

Official Record Copy

AFRL/RDLT

1 cy 